\title{
Management of tinea capitis in childhood
}

This article was published in the following Dove Press journal:

Clinical, Cosmetic and Investigational Dermatology

12 July 2010

Number of times this article has been viewed

\section{Antoni Bennassar \\ Ramon Grimalt \\ Dept of Dermatology, Hospital Clinic, University of Barcelona, Barcelona, Spain}

Correspondence: Ramon Grimalt Hospital Clinic,Villarroel I70, 08036

Barcelona, Spain

Fax +349378000I I

Email grimalt@ub.edu
Abstract: Tinea capitis (TC) is a common dermatophyte infection affecting primarily prepubertal children. The causative pathogens belong to only two genera: Trichophyton and Microsporum. Although there is a great local variation in the epidemiology of TC worldwide, T. tonsurans is currently the most common cause of TC with M. canis second. Even though there is an emerging number of anthropophilic scalp infections, $M$. canis remains the predominant causative organism in many countries of the Mediterranean basin, the most important dermatophyte carriers being stray cats and dogs as well as pet puppies, kittens and rabbits. TC always requires systemic treatment because topical antifungal agents do not penetrate down to the deepest part of the hair follicle. Since the late 1950s, griseofulvin has been the gold standard for systemic therapy of TC. It is active against dermatophytes and has a long-term safety profile. The main disadvantage of griseofulvin is the long duration of treatment required which may lead to reduced compliance. The newer oral antifungal agents including terbinafine, itraconazole, ketokonazole, and fluconazole appear to have efficacy rates and potential adverse effects similar to those of griseofulvin in children with TC caused by Trichophyton species, while requiring a much shorter duration of treatment. They may, however, be more expensive.

Keywords: tinea capitis, children, fungal infection, greseofulvin, terbinafine, itraconazole, fluconazole, treatment, pediatric infection

\section{Introduction}

Tinea capitis (TC) is a dermatophyte infection of the scalp hair follicles and intervening skin. ${ }^{1,2}$ It affects primarily prepubertal children. The reported prevalence in Europe is around $1.5 \%$.

The causative pathogens belong to only two genera: Trichophyton and Microsporum. Although there is a great local variation in the epidemiology of TC worldwide, T. tonsurans is currently the most common cause of TC with M. canis the second.

Even though there is an emerging number of anthropophilic scalp infections, $M$. canis remains the predominant causative organism in many countries of the Mediterranean basin, the most important dermatophyte carriers being stray cats and dogs as well as pet puppies, kittens and rabbits.

\section{Dermatophyte classification and pathogenesis of TC}

Dermatophytes are keratinophilic fungi which belong to three genera: Trichophyton, Microsporum, and Epidermophyton. On the basis of host preference and natural habitat, dermatophytes are classified as anthropophilic, zoophilic, and geophilic. 
TC is mainly caused by anthropophilic and zoophilic species of the genera Trichophyton and Microsporum. ${ }^{1,2}$

On the basis of the type of hair invasion, dermatophytes are also classified as endothrix, ectothrix or favus.

In endothrix infection the fungus grows completely within the hair shaft, the hyphae are converted to arthroconidia (spores) within the hair while the cuticle surface of the hair remains intact.

In ectothrix infection hair invasion develops in a manner similar to endothrix except that the hyphae destroy the hair cuticle and grow around the exterior of the hair shaft. Arthroconidia may develop both within and outside the hair shaft. Elongated hyphae, parallel to the long axis of the hair, persist within the hair.

Favus is characterized by production of hyphae, which are parallel to the long axis of the hair shaft. When the hyphae degenerate, long tunnels are left within the hair shaft. ${ }^{1,2}$

Ectothrix anthropophilic infections potentially spread rapidly whereas endothrix and favic infections are less contagious. $^{3}$

\section{Epidemiology}

TC is a common dermatophyte infection affecting primarily prepubertal children. Adults are infrequently affected. ${ }^{2,4,5}$ The reported prevalence in Europe ranges between 0.23\% and $2.6 \%{ }^{6,7}$

The causative pathogens belong to only two genera: Trichophyton and Microsporum. Although there is a great local variation in the epidemiology of TC worldwide, T. Tonsurans is currently the most common cause of TC and M. Canis the second one (Table 1). ${ }^{8}$

Even though there is an emerging number of anthropophilic scalp infections, $M$. canis remains the predominant causative organism in many countries of the Mediterranean basin, being stray cats and dogs as well as pet puppies, kittens and rabbits the most important dermatophyte carriers. ${ }^{8}$

On the other hand, anthropophilic TC has been mainly reported in children of Afro-Caribbean descent living in urban areas. These dermatophytosis are most frequently incurred by contact with an infected child, either directly

Table I Worldwide etiological agents causing TC

\begin{tabular}{ll}
\hline Species & Types \\
\hline Trichophyton tonsurans & Anthropophilic \\
Microsporum canis & Zoophilic \\
Microsporum audouinii & Anthropophilic \\
Trichophyton soudanense & Anthropophilic \\
Trichophyton violaceum & Anthropophilic \\
\hline
\end{tabular}

or via fomites. ${ }^{8}{ }^{8}$ It has been recently reported that an asymptomatic adult carrier may provide a source for continued reinfection in children.

T. schoenleinii causes a chronic form of TC that is usually acquired before adolescence and extending into adulthood. Fortunately, it has nearly disappeared from developed countries. ${ }^{10}$

\section{Clinical presentation}

Four clinical infection patterns have been reported. Different clinical presentation may arise depending on the causative organism, the type of hair invasion, and the specific host T-lymphocyte inflammatory response. ${ }^{4}$

Noninflammatory black dot pattern is clinically characterized by well-demarcated areas of hair loss. Fungal arthrospores proliferate inside the hair shafts, weakening them. Hairs break off at or below the scalp surface, giving the characteristic appearance of black dots on the alopecic patch. Cell-mediated immunity to fungal antigen skin test is usually negative and adenopathy is often absent. (Figure 1).

Noninflammatory seborrheic dermatitis type is a diffuse or patchy, fine, white, adherent scale affecting the scalp. This is the most difficult to diagnose because it resembles dandruff and only one third of patients have a positive potassium hydroxide examination.

In inflammatory tinea capitis (Kerion), there are one or multiple tender, inflamed, alopecic nodules with pustules on their surface. Fever, occipital adenopathy, leukocytosis, and even a diffuse, morbilliform rash may occur. Most patients have a positive skin test to fungal antigen, suggesting that the patient's immune response may account for the intense inflammation.

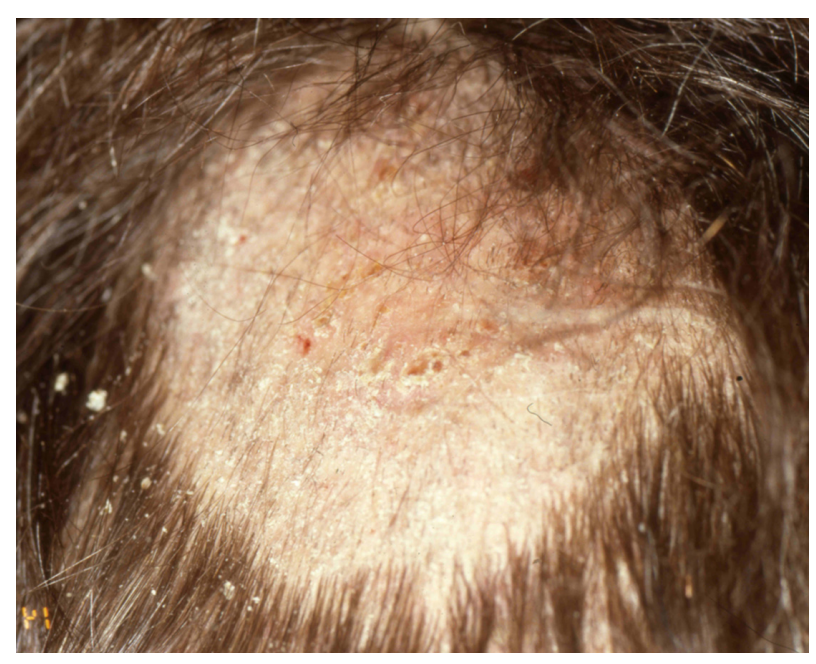

Figure I Tinea capitis endotrix. 
Favus is a rare type of inflammatory TC characterized by typical honey-colored, cup-shaped, follicular crusts called scutula. Kerion Celsi and favus have the potential to cause scarring and permanent alopecia (Figure 2). 2,3,11

A dermatophytid (id) reaction may accompany oral antifungal therapy and does not represent a widespread fungal infection. It is clinically characterized by a pruritic, papular or vesicular eruption that typically begins on the face and then spreads to the trunk. It is a reactive phenomenon that may be the result of a cell-mediated immune response to the dermatophyte, triggered by the antimycotic treatment. Topical steroids may be required to control the symptoms, but it is usually not necessary to discontinue oral antifungal treatment. Dermatophytid (id) reaction can also manifest as erythema nodosum. ${ }^{2}$

\section{Differential diagnosis}

Seborrheic dermatitis, atopic dermatitis, pseudotinea amiantacea and psoriasis may be confused with the diffuse noninflammatory form of TC. The black dot alopecia patterns should be differentiated from alopecia areata and trichotillomania whereas the inflammatory forms should be distinguished from bacterial infections or tumours. ${ }^{2,3,11}$ Tinea versicolor is usually very easy to differentiate.

\section{Asymptomatic dermatophyte scalp carriage}

The asymptomatic carrier state refers to a clinical situation where a person shows no signs or symptoms of TC, yet a

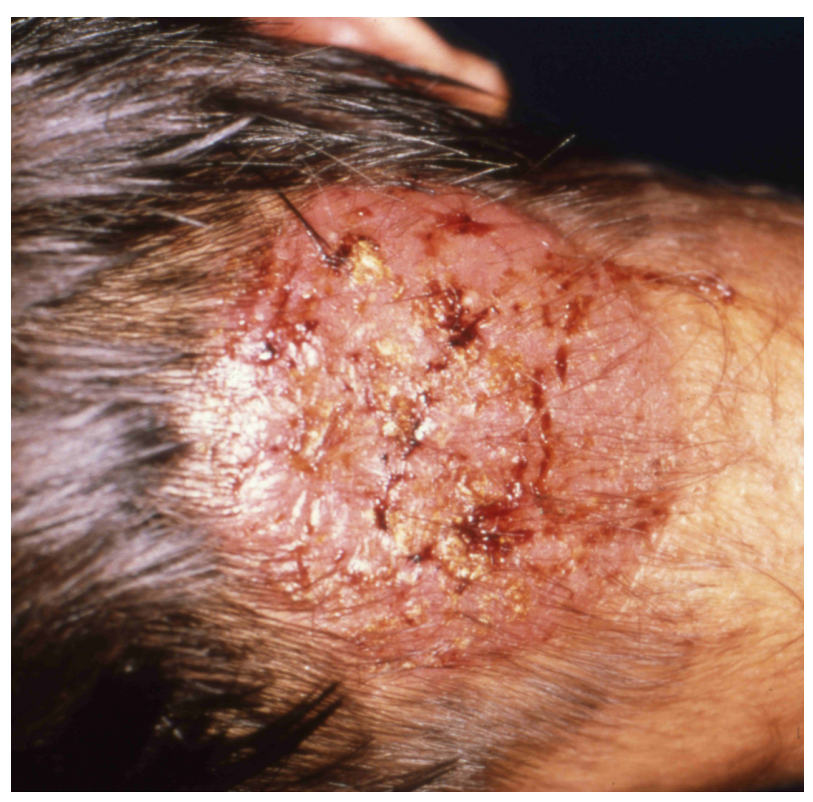

Figure 2 Kerion celsi. dermatophyte positive scalp culture is obtained. Although the asymptomatic carriage typically occurs in adults who have been exposed to children, it may also affect children. Asymptomatic carriers at home or at school shed the fungus and are potentially important sources of disease transmission. ${ }^{2,3}$ Consequently, some experts advocate treating all carriers with antimycotic drugs.

The aymptomatic carriage seems to be restricted to anthropophilic dermatophytes such as T. tonsurans, $T$. violaceum, and $M$. audouinii. These organisms generally lack host inflammatory response and consequently mild signs of infection escape clinical detection. On the other hand, zoophilic dermatophytes such as $M$. canis or T. mentagrophytes usually produce severe signs of infection and are, therefore, unlikely to lead to an asymptomatic carrier state.

\section{Laboratory diagnosis}

The laboratory diagnosis of scalp tinea is made by first examining scale and hair on a microscope slide in a potassium hydroxide wet mount and then culturing hair and scalp scale. Wood's light examination is of little value nowadays because in western countries most infections are due to T. tonsurans, which does not fluoresce.

\section{Specimen collection}

The specimen should be collected by experienced staff, in a sufficient amount, from the edge of the infected area, which corresponds to the active zone of the lesion.

Any crusts should be carefully removed with tweezers and the lesion should be disinfected with $70 \%$ alcohol before sampling, to remove contaminants such as bacteria. Due to electrostatic attraction, plastic boxes are unsuitable, so specimens have to be collected in sterile glass containers. ${ }^{12,13}$

Toothbrush culture sampling is largely of value for scaly lesions and consists of rubbing a sterilized toothbrush over the suspected areas.

For sampling an alopecic area with short hairs the most effective method is to rub the area firmly with a watermoistened tightly-woven gauze. Afterwards, each hair is lifted off the gauze with a needle or forceps and placed on a slide for potassium hydroxide preparation.

Human or animal asymptomatic carriers may be detected by rubbing the whole scalp or hair with a sterile piece of carpet, a sterile swab moistened with distilled water, or a hairbrush. The brush or carpet square are especially useful methods for suspected asymptomatic carrier cats or other pets. The brush or carpet square is combed through the coat, 
trapping fungal spores with hair and debris, and then pressed onto the surface of the culture medium. ${ }^{13}$

\section{Microscopic examination}

Direct microscopic examination of skin scrapings and hair is the most rapid method of establishing fungal etiology. Although it has been reported to have $5 \%-15 \%$ falsenegative results in routine practice, depending on the skill of the observer and on the quality of sampling, microscopic examination is essential, as it may allow the clinician to start treatment, pending culture results. ${ }^{13,14}$

Hair roots and skin scrapings are mounted in 10\%-20\% potassium hydroxide solution with or without dimethyl sulfoxide (DMSO). The slide is gently heated and microscopically examined for hyphae and spores. (Figure 3).

Other dissociating agents have also been proposed including Amann's chloral-lactophenol, which allows clearing without heating.

Congo red (a $b$-D-glucans stain) or Calcofluor white $0.1 \%$ solution (a chitin binding fluorochrome dye) added to the clearing reagent facilitate the visualization of fungal structures, but the latter requires use of a fluorescence microscope. ${ }^{13}$

The appearance of infected hairs depends on the invading dermatophyte species (Table 2). Hyphae must be differentiated from fibers of cotton wool or synthetic fabrics and from 'mosaic' which is a network of debris including cholesterol crystals around epidermal cells.

\section{Culture}

Plucked hair fragments and skin scrapings are placed directly on a culture medium. The brush-culture method involves gently rubbing a previously sterilized toothbrush in a circular motion over areas where scale is present, or over the margins

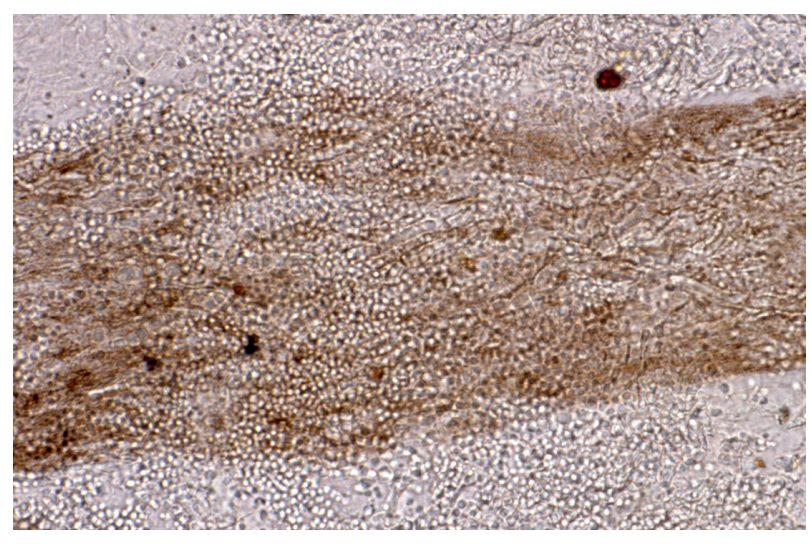

Figure 3 Optical examination of the infected hair shaft. Tinea capitis ectotrix.
Table 2 Hair invasion of dermatophytes

\begin{tabular}{ll}
\hline Site & Dermatophyte \\
\hline Ectothrix & M. audouinii \\
& M. canis \\
& M. ferrugineum \\
& T. mentagrophytes \\
Endothrix & T. verrucosum \\
& T. tonsurans \\
Favic & T. violaceum \\
\hline
\end{tabular}

of patches of alopecia. The brush fibers are then pressed into the culture media and the brush discarded. A cotton swab produces similar results. Cultures turn positive when using these collection techniques.

The two most common media are Sabouraud's agar and Mycobiotic agar containing chloramphenicol and cycloheximide to suppress the growth of bacterial saprophytic contamination. Dermatophyte test medium (DTM) is similar to Mycobiotic agar but contains a color indicator that changes from yellow to red in the presence of dermatophyte fungi.

Cultures are usually incubated at $20-30^{\circ} \mathrm{C}$ for $3-4$ weeks (or for up to 6 weeks if T. verrucosum, T. violaceum (Figure 4) or T. soudanense are suspected) and macroscopically screened at least twice a week for signs of fungal growth. Cultures usually show signs of growth in 7-10 days. Fungal identification is based on macroscopic (growth characteristics, pigment formation) as well as microscopic morphology (formation of macroconidia and microconidia or other typical elements). Additionally, in case of atypical isolates, some biochemical or physiological tests may be performed such as the search for urease activity or the in vitro hair perforation test. ${ }^{13}$

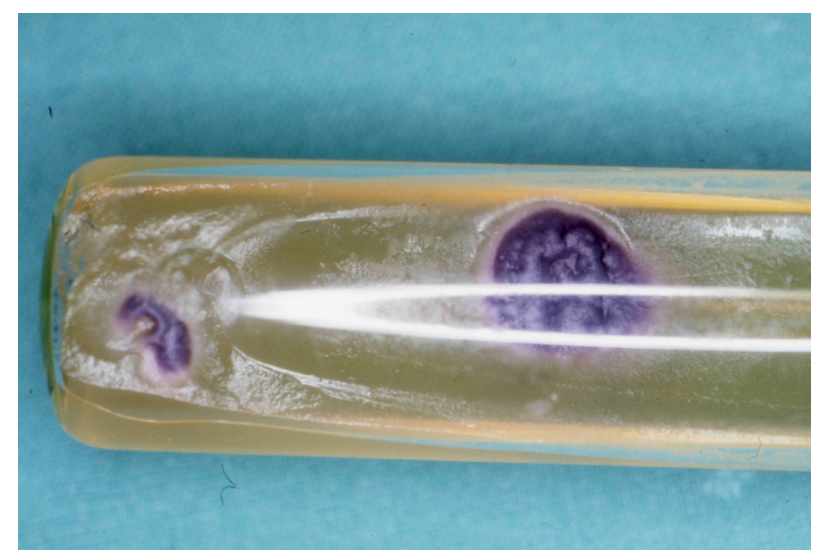

Figure $4 T$. Violaceum shows a highly characteristic aspect. 
Numerous methods for rapid nucleic acid-based distinction of dermatophyte species have also been described in recent years but are not routinely performed in clinical practice. ${ }^{15-17}$

\section{Wood's light examination}

The utility of Wood's ultraviolet light examination depends on whether the dermatophyte is an ectothrix or endothrix. When ectothrix dermatophytes such as $M$. canis, M. audouinii and $M$. distortum are examined under a Wood's lamp the hair shows a bright yellow-green fluorescence. ${ }^{18}$

Wood's light examination may, therefore, be a useful diagnostic aid for school screening surveys in ecthotrix anthropohilic cases.

On the other hand, endothrix dermatophytes like $T$. tonsurans and $T$. violaceum do not fluoresce at all and the use of Wood's light for screening and monitoring TC infection is limited.

\section{Treatment}

TC always requires systemic treatment because topical antifungal agents do not penetrate down to the hair follicle root. Topical treatment is only used as adjuvant therapy to systemic antifungals.

Factors that may influence the choice between equally effective therapies include tolerability, safety, compliance, availability of liquid formulation and cost.

Since the late 1950s, griseofulvin has been the gold standard for systemic therapy of TC. It is active against dermatophytes and has a long-term safety profile. The main disadvantage of griseofulvin is the long duration of treatment required (6-12 weeks or longer) which may lead to reduced compliance. ${ }^{19}$

The newer oral antifungal agents including terbinafine, itraconazole, ketokonazole, and fluconazole appear to have efficacy rates and potential adverse effects similar to those of griseofulvin in children with TC caused by Trichophyton species, while requiring a much shorter duration of treatment. They may, however, be more expensive. ${ }^{20}$ Consequently, the treatment decision between griseofulvin and newer antifungal agents for children with Trichophyton spp. tinea capitis can be based, for an individual patient, on the balance between duration of treatment/compliance and economic considerations. However terbinafin is preferably used to treat $T$. tonsurans tinea capitis in children, but it is not effective enough in the treatment of $M$. canis tinea capitis. On the other hand, fluconazole is more effective in the treatment of $M$. canis compared to terbinafine.
Conversely, griseofulvin is still the treatment of choice for cases caused by Microsporum species. Its efficacy is superior to that of terbinafine, ${ }^{21}$ and although its efficacy and treatment duration is matched by fluconazole ${ }^{22}$ and itraconazole ${ }^{23}$ griseofulvin is cheaper. It must be noted, however, that griseofulvin is nowadays not available in certain countries such as Belgium, Greece, Portugal, and Turkey.

Pending culture results, the choice of initial treatment should be based on the patient's history (eg, ethnic origin, contact with animals, practice of some particular sports), clinical presentation (eg, the black dot pattern of tinea capitis is commonly caused by $T$. tonsurans), direct microscopy (endothrix or ectothrix hair invasion) and compliance/cost.

It must be noted that country-specific prescribing information, and formula availability of any antifungal should be considered prior to prescription.

\section{Oral agents Griseofulvin}

Griseofulvin is fungistatic and inhibits the mitosis of dermatophytes by interacting with microtubules and disrupting the mitotic spindle; therefore it works best on actively-growing dermatophytes. It is fungistatic against Trichophyton, Microsporum and Epidermophyton. It is not active against yeasts (including Malassezia), dimorphic fungi causing deep infections, Cryptococcus or the fungi causing chromomycosis.

Griseofulvin has been available for more than 40 years and has proven to be safe. Two types of preparation are available: microsize and ultramicrosize, either in tablets or in oral suspension. The pediatric dose authorized for treating TC is $15-25 \mathrm{mg} / \mathrm{kg} /$ day using the microsize formulation. When the ultramicrosize formulation is used a dose of $10-15 \mathrm{mg}$ is recommended because it is better-absorbed than the microzise form. ${ }^{24}$ Griseofulvin produces a sustained blood level so it should be given in a single or in divided doses daily. Absorption varies from person to person: individual patients attain consistently high or low levels of drug. Taking the drug with fatty meals (eg, creamy yoghurt, chocolate or whole milk) may enhance absorption. The recommended duration of therapy for TC is 6-12 weeks or until the patient tests negative for fungi (light microscopy and culture). ${ }^{23,24}$ This long duration of treatment required with griseofulvin is a significant disadvantage and leads to noncompliance. As is the case with all systemic antifungals longer duration of treatment and a higher dose of griseofulvin is needed for ectothrix (eg, M. canis) than endothrix (eg, Trichophyton spp.) 
infections. Mycological cure and efficacy rates are generally high, being in the range of $80 \%-96 \%{ }^{25}$

Treatment failures can be observed due to poor compliance, fungal resistance, drug interactions, or side effects.

Griseofulvin is a safe drug. Headaches and gastrointestinal disturbances are the most common side effects. The dosage can be temporarily lowered to see if symptoms clear, but sometimes the drug must be discontinued. Severe allergic reactions, hepatic toxicity and leucopenia rarely occur; therefore routine blood studies are not necessary unless treatment is to last for many months or the dosage is exceptionally high (Table 3 ).

It is contraindicated in children with porphyria, lupus erythematosus, or severe liver disease. ${ }^{26}$

Drug interactions can occur with warfarin, phenobarbital and cyclosporine since griseofulvin is a potent inducer of microsomal cytochrome P-450 enzymes. ${ }^{24,25}$ The main disadvantage of griseofulvin is the long duration of treatment. ${ }^{25-28}$

\section{Terbinafine}

Terbinafine belongs to the allyamine class of drugs, a new generation of antifungal agents. It is fungicidal to dermatophytes since it inhibits squalene epoxidase, a membranebound enzyme in the biosynthetic pathway of sterol synthesis of the fungal cell membrane. It is well absorbed and binds strongly and nonspecifically to plasma proteins.

Table 3 Dosing pediatric regimens for the treatment of tinea capitis

\begin{tabular}{|c|c|c|}
\hline $\begin{array}{l}\text { Antifungal } \\
\text { agent }\end{array}$ & Dosage & $\begin{array}{l}\text { Duration of } \\
\text { treatment }\end{array}$ \\
\hline $\begin{array}{l}\text { Griseofulvin } \\
\text { Microsize } \\
\text { Ultramicrosize }\end{array}$ & $\begin{array}{l}20-25 \mathrm{mg} / \mathrm{kg} / \text { day } \\
10-15 \mathrm{mg} / \mathrm{kg} / \text { day }\end{array}$ & $\begin{array}{l}6-12 \text { weeks or } \\
\text { longer until fungal } \\
\text { cultures are } \\
\text { negative }\end{array}$ \\
\hline Terbinafine & $\begin{array}{l}10-20 \mathrm{~kg}: 62.5 \mathrm{mg} / \text { day } \\
20-40 \mathrm{~kg}: 125 \mathrm{mg} / \text { day } \\
>40 \mathrm{~kg}: 250 \mathrm{mg} / \mathrm{day} \\
\text { Or } \\
4-5 \mathrm{mg} / \mathrm{kg} / \text { day }\end{array}$ & $\begin{array}{l}\text { Trichophyton } \\
\text { spp.: } 2-4 \text { weeks } \\
\text { Microsporum spp.: } \\
8-12 \text { weeks }\end{array}$ \\
\hline Itraconazole & $\begin{array}{l}\text { Capsules: } 5 \mathrm{mg} / \mathrm{kg} / \mathrm{day} \\
\text { Oral solution: } 3 \mathrm{mg} / \mathrm{kg} / \mathrm{day}\end{array}$ & $\begin{array}{l}\text { Daily dosing: } \\
2-6 \text { weeks } \\
\text { Pulse regimen } \\
\text { (I week with } 2 \text { weeks } \\
\text { off between the first } \\
2 \text { pulses and } \\
3 \text { weeks between the } \\
2 \text { nd and } 3 \text { rd): } 2-3 \\
\text { pulses (range: I-5) }\end{array}$ \\
\hline Fluconazole & $\begin{array}{l}\text { Daily dosing: } 5-6 \mathrm{mg} / \mathrm{kg} / \text { day } \\
\text { Weekly dosing: } 8 \mathrm{mg} / \mathrm{kg} \\
\text { once weekly }\end{array}$ & $\begin{array}{l}3-6 \text { weeks } \\
8-12 \text { weeks }\end{array}$ \\
\hline
\end{tabular}

The absorption characteristics are not altered when terbinafine is taken with food. Its clearance in children is $40 \%$ higher than in adults. Since terbinafine is highly lipophilic and keratophilic, it is distributed throughout adipose tissue, dermis, epidermis, nails, and hair and persists in these tissues for weeks. Persistence of the drug in plasma is of concern when side effects are experienced. Terbinafine is delivered to the stratum corneum via the sebum and, to a lesser extent, through incorporation into the basal keratinocytes and diffusion through the dermis-epidermis. Terbinafine is not found in eccrine sweat. It remains in skin at concentrations above the mean inhibitory concentration (MIC) for most dermatophytes for 2 to 3 weeks after discontinuation of long-term oral therapy. After 6 and 12 weeks of oral therapy, terbinafine has been detected in the nail plate for 30 and 36 weeks, respectively, at a concentration well above the MIC for most dermatophytes. Terbinafine is metabolized in the liver, and dose adjustments may be needed in patients with liver or renal dysfunction.

It is available as $250 \mathrm{mg}$ tablets. The standard pediatric single daily dose is $62.5 \mathrm{mg}(10-20 \mathrm{~kg}) ; 125 \mathrm{mg}(20-40 \mathrm{~kg})$ and $250 \mathrm{mg}$ ( $\geq 40 \mathrm{~kg}$ ). Some suggest a weight-based dose of 4 to $5 \mathrm{mg} / \mathrm{kg}$ per day as an alternative. ${ }^{29}$ Terbinafine is concentrated in the hair and may remain present at fungicidal concentrations for several weeks after a course of treatment has been completed. ${ }^{30}$ The duration of treatment is generally 4 weeks, although shorter durations ( 2 weeks) have also been reported to be effective..$^{28,31,32}$

Higher dosages (10-25 kg: $125 \mathrm{mg} /$ day; >25 kg: $250 \mathrm{mg} /$ day or $12.5 \mathrm{mg} / \mathrm{kg} /$ day) or longer duration of treatment (8-12 weeks) may be required for $M$. canis infection. ${ }^{33-36}$

Side effects of terbinafine are rare and include gastrointestinal symptoms, rashes and headache. Liver enzyme abnormalities and drug reactions are occasionally seen. Plasma concentrations are reduced by rifampicin and increased by cimetidine. ${ }^{30}$

\section{Itraconazole}

Itraconazole is a triazole antifungal agent against Trichophyton and Microsporum spp. It exhibits both fungistatic and fungicidal activity depending on its concentration in the tissues, though its primary mode of action is fungistatic by inhibiting the cytochrome P-450-dependent enzymes, blocking the synthesis of ergosterol, the principal component of fungal cell membranes. Itraconazole is lipophilic and has a high affinity for keratinizing tissues. It adheres to the lipophilic cytoplasm of keratinocytes in the nail plate, allowing progressive buildup and persistence in the nail 
plate. The drug reaches high levels in the nails that persist for at least 6 months after discontinuation of 3 months of therapy and during pulsed cycles. The concentration in the stratum corneum remains detectable for 4 weeks after therapy. Itraconazole levels in sebum are 5 times higher than those in plasma and remain high for as long as 1 week after therapy. This fact suggests that secretion in sebum may account for the high concentrations found in skin. The drug has an affinity for mammalian cytochrome P-450 enzymes, as well as for fungal P-450-dependent enzyme, and thus has the potential for clinically-important interactions with astemizole, rifampicin, oral contraceptives, $\mathrm{H}_{2}$ receptor antagonists, warfarin and cyclosporine.

It is available as capsules or an oral solution. Itraconazole capsule formulation should be ingested with a meal whereas the oral solution should be taken in the fasting state for optimum bioavailability. The response to therapy does not appear to depend upon the formulation administered (capsules versus suspension).

The recommended pediatric dose is $5 \mathrm{mg} / \mathrm{kg}$ /day given continuously or by repeat pulsing. Where the oral solution is used, dosage is reduced to $3 \mathrm{mg} / \mathrm{kg} /$ day. $^{37}$

Using the continuous regimen, the duration of treatment for Trichophyton spp. ${ }^{38}$ and Microsporum spp. tinea capitis $^{39}$ is 2 and 6 weeks with cure rates of $85.7 \%$ and $88 \%$ respectively. It must be noted that the 6 -week regimen of itraconazole is of comparable efficacy to griseofulvin, in cases of Microsporum-TC. ${ }^{19}$

In the pulse regimen (one pulse of $5 \mathrm{mg} / \mathrm{kg} /$ day for 1 week with 2 weeks off between the first two pulses and 3 weeks between the second and third), the number of pulses required for the treatment depends in part on the severity of the TC..$^{40}$ In this way it may be possible to individualize the number of pulses administered according to the clinical response.

Side effects of itraconazole include headache, gastrointestinal complaints, rash and occasionally liver enzyme abnormalities. Less common is peripheral edema especially when taken with calcium channel blockers.

Itraconazole may increase plasma concentration of cyclosporine, certain benzodiazepines (midazolam, triazolam, alprazolam, and estazolam), digoxin, and cisapride. Concomitant use of $\mathrm{H}_{2}$-receptor antagonists, phenytoin, isoniazid, and rifampin may reduce the plasma concentration of itraconazole. Its use is strongly discouraged for patients with elevated or abnormal liver enzymes, active liver disease or who have experienced liver toxicity with other antifungal azole drugs. It is contraindicated in patients with evidence of ventricular dysfunction such as congestive heart failure.

\section{Fluconazole}

Fluconazole is primarily a fungistatic triazole, preventing the conversion of lanosterol to ergosterol, an essential component of the fungal cytoplasmic membrane. It is distinguished from the other azoles by its water solubility that results in excellent bioavailability by the oral route. Because fluconazole is highly soluble in water it is transported to the skin through sweat and concentrated by evaporation. It achieves high concentrations in the epidermis and nails and persists up to 3 months. ${ }^{41}$

It is available as a tablet or oral suspension. Doses of 5-6 mg/kg/ per day for 4-6 weeks can effectively treat TC. ${ }^{22}$ Once-weekly $8 \mathrm{mg} / \mathrm{kg}$ pulse dosing for $8-12$ weeks is an alternative regimen. ${ }^{42}$

Evidence suggest that in respect to Trichophyton species TC, a 2-4-week regimen of fluconazole has similar cure rates to a 6-week regimen of griseofulvin. ${ }^{20}$

Two studies that included 140 children found similar cure rates of 2-4 weeks of fluconazole when compared with 6 weeks of griseofulvin (RR $0.92 ; 95 \%$ CI 0.80 to 1.05 ).

Side effects of fluconazole are similar to other azole derivatives. Hematologic and hepatic toxicity may occasionally occur.

Drug interaction: terfenadine, cisapride (risk of serious cardiac arrhythmias)

Contraindications: severe liver disease. Use with caution in patients sensitive to other azoles.

\section{Topical agents}

Adjunctive topical therapies such as Selenium sulphide, ${ }^{43}$ zinc pyrithione, povidoneiodide or ketoconazole ${ }^{44}$ shampoos as well as fungicidal creams or lotions ${ }^{45}$ have been shown to decrease the carriage of viable spores responsible for the disease contagion and reinfection and may shorten the cure rate with oral antifungal. A terbinafine solution $0.01 \%$ completely killed arthroconidia of five Trichophyton species after an exposure time of 15-30 min. ${ }^{46}$

The topical fungicidal cream/lotion should be applied to the lesions once daily for a week. ${ }^{45}$

The shampoo should be applied to the scalp and hair for 5 minutes twice-weekly for 2-4 weeks ${ }^{39,47}$ or three times weekly until the patient is clinically and mycologically cured. ${ }^{19}$ The authors recommend the latter in conjunction with one week of topical fungicidal cream or lotion application.

\section{Additional measures School attendance}

Keeping children out of school after starting therapy is controversial. Several experts suggest that once treatment 
has been initiated with oral and topical agents, the children should, for practical reasons, be allowed back to school or day care although there is still a risk of infecting fellow students. ${ }^{26}$ On the other hand, other experts recommend exemption from school/kindergarten attendance, regardless of the type of dermatophyte, for approximately 2 weeks after initiation of treatment, a period necessary for significant decline of infection load in the hair follicle. ${ }^{45}$

Patient education is, therefore, of utmost importance in eradicating $\mathrm{TC}$.

It must be stressed that the degree of disease transmission depends on the type of the dermatophyte isolated, the most contagious being the ectothrix anthropophilic. The latter potentially spreads rapidly and often causes epidemics in schools. ${ }^{48}$ Additionally, topical fungicidal treatment, nowadays, can kill arthroconidia rapidly. ${ }^{46}$

Therefore the following are recommended: If the causative agent is an anthropophilic ectothrix the child should usually be allowed school/kindergarten attendance one week after initiation of treatment. Wood's light is useful to monitor the disappearance of contaminating spores. In all other cases the child should be allowed to attend school/kindergarten as soon as the treatment has been initiated.

When the child is back at school, he/she should be strongly advised not to share items such as combs, hairbrushes, scarves, and hats, as fomites may play a role in transmission. School staff may help in enforcing this.

So, in all cases with TC caused by anthropophilic dermatophytes the school authorities should be notified. ${ }^{48}$

Sports that lead to prolonged close physical contact (eg, wrestling) should be prohibited until the risk of infection no longer exists.

Plucking the involved hair, as practiced in many countries, may help in the rapid resolution of the infection as it physically removes large amounts of yeasts.

\section{Sources of infection}

Upon positive microscopy and pending culture clinical examination of family members is urgently recommended. Appropriate mycological samples should be taken initially only from those with signs of infection.

Zoophilic organisms such as $M$. canis cause an inflammatory response in nearly all those infected. Conversely, anthropophilic organisms, usually either $T$. tonsurans or $T$. violaceum cause a mild or noninflammatory response, thus making them good candidates for asymptomatic carriage. ${ }^{2}$ Subsequently, if an anthropophilic organism is finally identified by culture in the index case, then appropriate quantitative culturing should be performed on all family members/close contacts even in the absence of clinical signs (brush method). 'Close contacts' include playmates in close physical contact, and additionally, in very young children (kindergarten through second grade) their schoolmates, since these children are more susceptible and have a greater risk of disease transmission.

It remains unclear whether carriers should be treated with topical antifungal shampoos or oral antifungal, with both, or with neither. In those with moderate or heavy growth of culture, oral therapy may be justified as these individuals are particularly likely to develop an overt clinical infection; they are a reservoir of transmission, and are unlikely to respond to topical treatment alone.

For those with low spore counts on culture, twice-weekly selenium sulfide or $2 \%$ ketoconazole shampoo for up to 12 weeks is probably adequate. ${ }^{3}$

Pets (eg, dogs, cats, guinea pigs, hamsters) should also be examined and treated as necessary.

It must be noted, however, that stray cats or dogs frequently infect children living in developing countries.

Viable fungal spores have been isolated from the floor, backs of chairs, clothing, beds, pillows, curtains, brushes, combs, scissors, and other shared facilities in the household. Consequently the washable items (eg, bedding and textiles) should be laundered, carpets should be vacuum-cleaned, and floors mopped with a strong disinfectant. Brushes and combs as well as other hair accessories should be disinfected after use or discarded. ${ }^{3}$ The 2007 German-Speaking Mycological Society Guideline on TC noted that for items that can be boiled, eg, combs or possibly hairbrushes, $5 \mathrm{~min}$ in boiling water is sufficient. Scissors may be placed in an instrument disinfectant eg, $5 \mathrm{~min}$ in a Mucocit-B drill bath (this alcoholbased product is designed for disinfecting dental drills). ${ }^{45}$

\section{Steroids/antibiotics/antihistamines}

Current data indicate that the use of steroids for Kerion Celsi may reduce scaling and itching but does not reduce the clearance time compared with griseofulvin alone. ${ }^{49,50}$ Prednisolone may be used as oral treatment at $1 \mathrm{mg} / \mathrm{kg}$ per day for 7 days though this is not recommended as part of routine care for kerion. ${ }^{51}$

Also, there are no studies that support the routine use of antibiotics in patients with kerion because kerion Celsi is rarely subject to secondary bacterial infection. ${ }^{52}$ Incision or excision of kerion nodules is not recommended. ${ }^{53}$

In patients with pruritus, systemic antihistamines can reduce discomfort and may prevent distribution of spores via finger scratching. 


\section{Follow-up}

Clinical and mycological examinations of affected children should be conducted at regular intervals ( $2-4$ weeks). The treatment may be stopped after the culture becomes negative or when hair regrowth is clinically evident: consequently the duration of treatment can be individualized according to the response.

The causes of treatment failure include suboptimal absorption of the medication, relative insensitivity of the organism, reinfection and lack of compliance with the long courses of treatment.

If at the end of the standard treatment period fungi can still be isolated from the lesional skin, but clinical signs have improved, the recommendation is to continue the original regimen for another month. If there has been no clinical improvement then, the original regimen can again be extended for a further month though in these cases it is also reasonable to switch to an alternative antifungal. Periodic monitoring of hepatic enzymes and complete blood count is recommended in children during prolonged therapy with itraconazole or terbinafine ( $>4$ and 6 weeks, respectively). ${ }^{19}$ Additionally renal function should be monitored when the child is receiving prolonged treatment with griseofulvin or fluconazole.

\section{Complications}

Some complications have already been cited in the text but we should also consider the possibility of scarring, cicatricial alopecia, superinfections by bacteria (impetigo) and changes in skin color.

If the treatment is adequate, in general, the prognosis is good.

\section{Conclusion}

We believe that most studies indicate that there is enough evidence to support the use of griseofulvin to treat tinea capitis in children, which is caused by T. tonsurans, M. canis, T. mentagrophytes, and T. violaceum.

Overall, griseofulvin is considered to be safe in children.

Terbinafine, when compared with griseofulvin, produces good results in a shorter time of treatment, making participant compliance less of a problem.

One potential disadvantage, however, is that terbinafine is only available in tablet form. While tablets may be preferred by some children (aged five years and older, perhaps), they may not allow for dosage individualization.

We believe that although griseofulvin will continue to remain the antifungal drug of choice in tinea capitis, terbinafine may constitute an alternative drug which is well tolerated and has few side effects. It would be interesting to see more comparisons between the newer and relatively expensive antifungals for tinea capitis in children. There are currently a limited number of trials involving different doses, and further information is needed on treatment doses and frequency for all antifungals including griseofulvin.

\section{Acknowledgments}

Images 1 to 4 are courtesy of Dr M Lecha and Dr V Lecha.

\section{Disclosure}

The authors report no conflicts of interest in this work.

\section{References}

1. Gupta AK, Summerbell RC. Tinea capitis. Med Mycol. 2000; 38:255-287.

2. Elewski B. Tinea capitis: a current perspective. J Am Acad Dermatol. 2000;42:1-20

3. Ilkit M, Demirhindi H. Asymptomatic dermatophyte scalp carriage: laboratory diagnosis, epidemiology and management. Mycopathologia. 2008;165:61-71.

4. Razzaq Adel AA, Sultan AO, Basmiah AM, Aftab A, Nabel N. Prevalence of tinea capitis in southern Kuwait. Mycoses. 2007;50:317-320.

5. Romano C, Gianni C, Papini M. Tinea capitis in infants less than 1 year of age. Pediatr Dermatol. 2001;18:465-468.

6. Trivino-Duran L, Torres-Rondriguez JM, Martinez-Roig A, et al. Prevalence of tinea capitis and tinea pedis in Barcelona schoolchildren. Pediatr Infect Dis J. 2005;24:137-141.

7. Hay RJ, Clayton YM, De Silva N, Midgley G, Rossor E. Tinea capitis in south-east London: a new pattern of infection with public health implications. Br J Dermatol. 1996;135:955-958.

8. Hay RJ, Robles W, Midgley G, Moore MK. European Confederation of Medical Mycology Working Party on Tinea Capitis. Tinea capitis in Europe: new perspective on an old problem. J Eur Acad Dermatol Venereol. 2001;15:229-233.

9. Ginter-Hanselmayer G, Weger W, Ilkit M, Smolle J. Epidemiology of tinea capitis in Europe: current state and changing patterns. Mycoses. 2007;50 Suppl 2:6-13.

10. Niczyporuk W, Krajewska-Kuzak E, Zukaszuk C. Tinea capitis favosa in Poland. Mycoses. 2004;47:257-260.

11. Fuller LC, Child FJ, Midgley G, Higgins EM. Diagnosis and management of scalp ringworm. BMJ. 2003;326:539-541.

12. Boralevi F, Léauté-Labrèze C, Roul S, Couprie B, Taïeb A. Lupuserythematosus-like eruption induced by Trichophyton mentagrophytes infection. Dermatology. 2003;206:303-306.

13. Robert R, Pihet M. Conventional methods for the diagnosis of dermatophytes. Mycopathologia. 2008;166:295-306.

14. Panasiti V, Borroni RG, Devirgiliis V, et al. Comparison of diagnostic methods in the diagnosis of dermatomycoses and onychomycoses. Mycoses. 2006;49:26-29.

15. Liu D, Coloe S, Baird R, Pedersen J. Application of PCR to the identification of dermatophyte fungi. J Med Microbiol. 2000;49:493-497.

16. Kac G. Molecular approaches to the study of dermatophytes. Med Mycol. 2000;38:329-336.

17. Kanbe T, Suzuki Y, Kamiya A, et al. Species identification of dermatophytes Trichophyton, Microsporum and Epidermophyton by PCR and PCR-RFLP targeting of the DNA topoisomerase II genes. J Dermatol Sci. 2003;33:41-54. 
18. Kefalidou S, Odia S, Gruseck E, Schmidt T, Ring J, Abeck D. Wood's light in Microsporum canis positive patients. Mycoses. 1997;40:461-463.

19. Elewski BE. Treatment of tinea capitis: beyond griseofulvin. JAm Acad Dermatol. 1999;40(6 Pt 2):S27-S30.

20. Gonzalez U, Seaton T, Bergus G, Jacobson J, Martinez-Monzon C. Systemic antifungal therapy for tinea capitis in children. Cochrane Database Sys Rev. 2007;17:CD004685.

21. Elewski BE, Cáceres HW, DeLeon L, et al. Terbinafine hydrochloride oral granules versus griseofulvin suspension in children with tinea capitis: results of two randomized, investigator-blinded, multicenter, international, controlled trials. J Am Acad Dermatol. 2008;59:41-54.

22. Foster KW, Friedlander SF, Panzer H. A randomized controlled trial assessing the efficacy of fluconazole in treatment of pediatric tinea capitis. J Am Acad Dermatol. 2005;53:798-809.

23. López-Gómez S, Del Palacio A, Van Cutsem J, Soledad Cuétara M, Iglesias L, Rodriguez-Noriega A. Itraconazole versus griseofulvin in the treatment of tinea capitis: a double-blind randomized study in children. Int J Dermatol. 1994;33:743-747.

24. Roberts BJ, Friedlander SF. Tinea capitis: a treatment update. Pediatr Ann. 2005;34:191-200.

25. Bennett ML, Fleisher AB, Loveless JW, Feldman SR. Oral griseofulvin remains the treatment of choice for tinea capitis in children. Pediatr Dermatol. 2000;17:304-309.

26. Higgins EM, Fuller LC, Smith CH. Guidelines for the management of tinea capitis. British Association of Dermatologists. Br J Dermatol. 2000;143:53-58.

27. Gupta AK, Cooper EA, Bowen JE. Meta-analysis: griseofulvin efficacy in the treatment of tinea capitis. J Drugs Dermatol. 2008;7:369-372.

28. Gupta KA, Adam P, Dlova N, et al. Therapeutic options for the treatment of tinea capitis caused by trychophyton species: Griseofulvin versus the new oral antifungal agants, terbinafine, itraconazole and fluconazole. Pediatr Dermatol. 2001;18:433-438.

29. Gupta AK, Adamiak A, Cooper EA. The efficacy and safety of terbinafine in children. J Eur Acad Dermatol Venereol. 2003;17:627-640.

30. Gupta AK, Cooper EA, Lynde CW. The efficacy and safety of terbinafine in children. Dermatol Clinics. 2003;21:511-520.

31. Friedlander SF, Aly R, Krafchik B, et al. A randomized, doubleblind, parallel group duration-finding study of oral terbinafine in children with tinea capitis due to Trichophyton species. Pediatr. 2002;109:602-607.

32. Haroon TS, Hussain I, Aman S, et al. A randomized double-blind comparative study of terbinafine for 1,2 and 4 weeks in tinea capitis. Br J Dermatol. 1996;135:86-88.

33. Lipozencic J, Skerlev M, Orofino-Costa R, et al. A randomized, doubleblind, parallel-group, duration-finding study of oral terbinafine and open-label, high-dose griseofulvin in children with tinea capitis due to Microsporum species. Br J Dermatol. 2002;146:816-823.

34. Koumantaki E, Kakourou T, Rallis E, Riga P, Georgalla S. Doubled dose of oral terbinafine is required for Microsporum canis tinea capitis. Pediatr Dermatol. 2001;4:339-342.

35. Friedlander SF, Aly R, Krafchik B, et al. Terbinafine in the treatment of Trichophyton tinea capitis: a randomized, double-blind, parallel-group, duration-finding study. Pediatr. 2002;109:602-607.
36. Devliotou-Panagiotidou D, Koussidou-Eremondi TH. Efficacy and tolerability of 8 weeks' treatment with terbinafine in children with tinea capitis caused by Microsporum canis: a comparison of three doses. J Eur Acad Dermatol Venereol. 2004;18:155-159.

37. Gupta AK, Solomon RS, Adam P. Itraconazole oral solution for the treatment of tinea capitis. Br J Dermatol. 1998;139:104-106.

38. Jahangir M, Hussain I, Ul Hasan M, Haroon TS. A double-blind, randomized, comparative trial of itraconazole versus terbinafine for 2 weeks in tinea capitis. Br J Dermatol. 1998;139:672-674.

39. Ginter-Henselmayer G, Smolle J, Gupta A. Itraconazole in the treatment of tinea capitis caused by Microsporum canis: Experience in a large cohort. Pediatr Dermatol. 2004;21:499-502.

40. Gupta AK, Hofstader SL, Summerbell RC, et al. Treatment of tinea capitis with itraconazole capsule pulse therapy. $J$ Am Acad Dermatol. 1998;39(2 Pt 1):216-219.

41. Dastghaib L, Azizzadeh M, Jafari P. Therapeutic options for the treatment of tinea capitis: Griseofulvin versus fluconazole. J Dermatol Treat. 2005; $16: 43-46$.

42. Gupta AK, Dlova N, Taborda P, et al. Once-weekly fluconazole is effective in children in the treatment of tinea capitis: a prospective, multicentre study. Br J Dermatol. 2000;142:965-968.

43. Allen HB, Honig PJ, Leyden JJ, McGinley KJ. Selenium sulfide: adjunctive therapy for tinea capitis. Pediatrics. 1982;69:81-83.

44. Greer DL. Successful treatment of tinea capitis with $2 \%$ ketoconazole shampoo. Int J Dermatol. 2000;39:302-304.

45. Seebacher C, Abeck D, Brasch J, et al. Tinea capitis: ringworm of the scalp. Mycoses. 2007;50:218-226.

46. Gupta AK, Ahmad I, Summerbell RC. Comparative efficacies of commonly used disinfectants and antifungal pharmaceutical spray preparations against dermatophytic fungi. Med Mycol. 2001;39:321-328.

47. Fuller LC, Smith CH, Cerio R, et al. A randomised comparison of four weeks of terbinafine versus eight weeks of griseofulvin for the treatment of tinea capitis - advantages of a shorter treatment schedule. Br J Dermatol. 2001;144:321-327.

48. Weill FX, Bernier V, Maleville J, et al. Epidémie de teignes du cuir chevelu à microsporum audouinii var. langeronii dans un groupe scholaire. Bordelais J Mycol Méd. 1999;9:52-56.

49. Honig PJ, Caputo GL, Leyden JJ, McGinley K, Selbst SM, McGravey AR. Treatment of kerions. Pediatr Dermatol. 1994;11:69-71.

50. Hussain I, Muzaffar F, Rashid T, Ahmad TJ, Jahangir M, Haroon TS. A randomized, comparative trial of treatment of kerion celsi with griseofulvin plus oral prednisolone vs griseofulvin alone. Med Mycol. 1999;37:97-99.

51. Ali S, Graham TA, Forgie SE. The assessment and management of tinea capitis in children. Pediatr Emerg Care. 2007;23:662-665.

52. Thoma-Greber E, Zenker S, Röcken M, Wolff H, Korting HC. Surgical treatment of tinea capitis in childhood. Mycoses. 2003;46:351-354.

53. von Laer Tschudin L, Laffitte E, Baudraz-Rosselet F, Dushi G, Hohlfeld J, de Buys Roessingh AS. Tinea capitis: no incision nor excision. J Pediatr Surg. 2007;42:E33-E36.
Clinical, Cosmetic and Investigational Dermatology

\section{Publish your work in this journal}

Clinical, Cosmetic and Investigational Dermatology is an international, peer-reviewed, open access, online journal that focuses on the latest clinical and experimental research in all aspects of skin disease and cosmetic interventions. All areas of dermatology will be covered; contributions will be welcomed from all clinicians and

\section{Dovepress}

basic science researchers globally. This journal is indexed on CAS The manuscript management system is completely online and includes a very quick and fair peer-review system, which is all easy to use. Visit http://www.dovepress.com/testimonials.php to read real quotes from published authors. 